\title{
Three-phase fractionation of polydisperse fluids
}

\author{
L. Bellier-Castella ${ }^{\dagger}$, M. Baus ${ }^{\dagger \dagger}$ and H. Xu ${ }^{\dagger}$ \\ (April 24, 2001) \\ † Département de Physique des Matériaux (UMR 5586 du CNRS), \\ Université Claude Bernard-Lyon1, 69622 Villeurbanne Cedex, France \\ †† Physique des Polymères, Université Libre de Bruxelles, \\ Campus Plaine, CP 223, B-1050 Brussels, Belgium
}

PACS numbers: 05.70.-a, 64.75.+g, 82.70.-y 


\begin{abstract}
It is shown that the van der Waals free-energy of polydisperse fluids, as introduced previously ( L. Bellier-Castella, H. Xu and M. Baus, J. Chem. Phys. 113, 8337 (2000) ), predicts that for certain thermodynamic states (e.g. low temperatures and large polydispersities) the ordinary two-phase coexistences become metastable relative to a fractionation of the system into three phases, reducing thereby the polydispersity of each of the coexisting phases.
\end{abstract}




\section{INTRODUCTION}

A polydisperse system can be viewed as a "continuous mixture", i.e. as the limit of a discrete mixture whereby the number of component species tends to infinity while the concentration of each species tends to zero 1 . Such a system serves as a good model for many of the complex fluids used in various industries 1 . Whereas many of these industrial fluids have badly characterized concentration distributions one can find at present, e.g. in the physics laboratories, several soft condensed matter systems 3 (e.g. colloids, liquid crystals, polymers, etc) with much better controled species distributions allowing hence for a more detailed study of the influence of the polydispersity on various physical properties (e.g. phase behavior, rheology, etc.). To transpose the results and techniques elaborated for simple fluids and their mixtures to polydisperse or continuous mixtures is however by no means a simple task but has nevertheless recently become an active field of research - -

In the present study, a sequel to ref.8, we will be concerned with the equilibrium phase behavior of polydisperse fluids. To simplify the problem, we restrict ourselves to systems of spherical particles (e.g. colloids) exhibiting a single polydispersity, say a size-polydispersity. We assume moreover the initial (parent phase) size-distribution to be monomodal, i.e. centered around a single reference species, as suitable for the polydisperse generalization of one-component systems. This initial size distribution will further be assumed to be fixed, once and for all, by the production process of, say, the colloidal particles. (Note that some systems, e.g. micellar solutions, exhibit a "variable" polydispersity whereby the size distribution is allowed to adjust itself to some externally imposed conditions 9 .) When these colloidal particles interact by excluded volume repulsions and, say, van der Waals (vdW) attractions their thermodynamic properties can be studied on the basis of the polydisperse generalization of the vdW free-energy 10 , such as the one already used in our earlier studiest- 8 . Of course, such a description is far from being exact, but in general the vdW-approximation captures, at least qualitatively, the essence of the underlying phase behavior, as can be witnessed from several previous investigations11. As a final limitation, we would like to stress that our study will be limited to the fluid phases of the polydisperse system, leaving aside whether these phases are stable or metastable with respect to the possible solid phases. Indeed, the inclusion of the solid phases would require a more precise specification of the interaction potential than is usual in a vdW-description and, above all, would add still more complexity to the already fairly complex problems raised by the study of phase equilibria in polydisperse fluids. In this way, we will be able to focus on the central difficulty resulting from the replacement of the algebraic equations in finite dimensional spaces, characteristic of the phase behavior of discrete mixtures, by the integral equations in infinite dimensional spaces characteristic of the continuous mixtures.

In our previous study, 8 , the phase behavior of the present system was already studied within the same vdW-approximation, but only at the level of the two-phase coexistences. It was shown there that the polydispersity can have a profound influence on the binodals of this system as compared with its monodisperse counterpart. One aspect which was not studied in ref.8 concerns the fact that, as a consequence of the Gibbs phase rule, a polydisperse system can also phase separate or "fractionate" into more than two fluid phases, 
each phase differing both in average density and in size distribution. The study of such a polydispersity-induced equilibrium between three fluid phases is the main object of the present investigation. Meanwhile, we will also consider another question left unanswered in ref.8. This concerns the specific form given to the parent-phase size-distribution. In ref.8 we used some well-known expressions (Schulz-Zimm and log-normal) for this input size-distribution. These theoretical distributions describe particles with sizes ranging from zero to infinity. Since both the very small and very large particles are absent from the experimentally studied systems, one may wonder whether their presence in these theoretical expressions could not lead to artifacts in the phase diagram. The influence of these tails (for both small and large sizes) of the theoretical distributions on the coexistence properties of ref. 8 will hence be investigated here before proceeding to the three-phase equilibria.

In section [II we recall the vdW-description of polydisperse fluids as used in ref.8. Section III is devoted to the influence of the tails of the parent-phase distribution on the results of ref.8. In section $\mathbb{I V}$ we show that the present vdW-description allows for the phase equilibrium between three fluid phases and we study the relative stability of the two-phase and three-phase equilibria in section $\nabla$. Our conclusions are given in the final section $\nabla \mathbb{1}$.

\section{THE POLYDISPERSE VDW FREE-ENERGY AND ITS CRITICAL POINTS}

The thermodynamic properties of a fluid of spherical particles with a size-distribution can be determined from the following generalization of the vdW free-energy (see ref.8 for details):

$$
\begin{array}{rl}
f(T,[\rho])=k_{B} & T \int d \sigma \rho(\sigma)\left\{\ln \left(\frac{\Lambda^{3}(\sigma) \rho(\sigma)}{E[\rho]}\right)-1\right\} \\
+ & \frac{1}{2} \int d \sigma \int d \sigma^{\prime} V\left(\sigma, \sigma^{\prime}\right) \rho(\sigma) \rho\left(\sigma^{\prime}\right)
\end{array}
$$

where, $f(T,[\rho])$, is the free-energy per unit volume at the temperature $T\left(k_{B}\right.$ being Boltzmann's constant) for a fluid for which the number density of species $\sigma$ is $\rho(\sigma)$, while $\Lambda(\sigma)$ denotes the thermal de Broglie wavelength of species $\sigma$. Any functional dependence on the density distribution $\rho(\sigma)$ is, as usual, indicated by $[\rho]$. In (1), the excluded volume repulsions are represented by:

$$
E[\rho]=1-\int d \sigma v(\sigma) \rho(\sigma)
$$

where, $v(\sigma)=\frac{4 \pi}{3}(R(\sigma))^{3}$, is the volume of a spherical particle of radius $R(\sigma)$, whereas the attractions are represented by:

$$
V\left(\sigma, \sigma^{\prime}\right)=\int d \mathbf{r} V_{A}\left(r ; \sigma, \sigma^{\prime}\right)
$$

with $V_{A}\left(r ; \sigma, \sigma^{\prime}\right)$ being the potential of attraction between two particles of species $\sigma$ and $\sigma^{\prime}$, a distance $r=|\mathbf{r}|$ apart. In the above $\sigma$ represents both a species label and the dimensionless polydispersity variable, $R(\sigma) / R(1), R(1)$ being the radius of the reference species, $\sigma=1$.

The pressure, $p(T,[\rho])$, corresponding to (11) is: 


$$
p(T,[\rho])=\frac{k_{B} T}{E[\rho]} \int d \sigma \rho(\sigma)+\frac{1}{2} \int d \sigma \int d \sigma^{\prime} V\left(\sigma, \sigma^{\prime}\right) \rho(\sigma) \rho\left(\sigma^{\prime}\right) .
$$

whereas the chemical potential of species $\sigma, \mu(\sigma, T,[\rho])$, reads:

$$
\begin{aligned}
\mu(\sigma, T,[\rho])=k_{B} T \ln \left\{\frac{\Lambda^{3}(\sigma) \rho(\sigma)}{E[\rho]}\right\} & +k_{B} T \frac{v(\sigma)}{E[\rho]} \int d \sigma^{\prime} \rho\left(\sigma^{\prime}\right) \\
& +\int d \sigma^{\prime} V\left(\sigma, \sigma^{\prime}\right) \rho\left(\sigma^{\prime}\right) .
\end{aligned}
$$

In the above, all integrals over $\sigma$ extend over the whole domain for which $\rho(\sigma)$ is non-zero. As in ref.8, we write, $\rho(\sigma)=\rho h(\sigma)$, where $\rho=\int d \sigma \rho(\sigma)$ is the average density and $h(\sigma)$ the (normalized) size-distribution. The dimensionless average density will be written as, $\eta=\rho v(1)$, with $v(1)$ the volume of the reference species $\sigma=1$, while the dimensionless temperature will be, $t=k_{B} T / \epsilon(1,1)$, with $\epsilon(1,1)=-V(1,1) / 8 v(1)$ and $V(1,1)$ being the integrated amplitude of the attraction between two reference particles (cf.(3) and ref.8).

In ref.8 we have considered several types of polydisperse interactions characterized by two parameters $\{l, n\}$, viz. $v(\sigma)=v(1) \sigma^{3 n}$ and $V\left(\sigma, \sigma^{\prime}\right)=\left[\left(\sigma^{n}+\sigma^{\prime n}\right) / 2\right]^{3}\left(\sigma \sigma^{\prime}\right)^{l} V(1,1)$. As shown there the model with $\{l=1, n=0\}$ has a phase behavior which is similar to that with the full polydispersity $\{l=1, n=1\}$ but is simpler to study because its excess free-energy involves fewer moments, $m_{k}=\int d \sigma \sigma^{k} h(\sigma)$, of the size-distribution $h(\sigma)$. Henceforth, we will therefore consider only this $\{l=1, n=0\}$-model, so as to simplify the calculations. In ref.8 we also considered several types of parent-phase size-distributions $h_{0}(\sigma)$ but found no qualitative differences between them. In order to avoid too much duplication of results, we will henceforth consider only the log-normal parent-phase size-distribution, $h_{0}(\sigma)=c \exp \left[-a \ln ^{2}(\sigma / b)\right]$, where the three parameters $\{a, b, c\}$ are determined by imposing the first three moments of $h_{0}(\sigma), m_{k}^{(0)}=\int_{0}^{\infty} d \sigma \sigma^{k} h_{0}(\sigma)$, to be such that: 1) $h_{0}(\sigma)$ be normalized $\left.\left(m_{0}^{(0)}=1\right), 2\right)$ the average value of $\sigma$ be one, i.e. equal to the reference species $\left.\left(m_{1}^{(0)}=1\right), 3\right)$ the variance of $h_{0}(\sigma)$ be $\frac{1}{\alpha}=I-1>0$, with $I$ being the polydispersity index $\left(m_{2}^{(0)}=I\right)$. In terms of $I$ this yields: $a=1 / 2 \ln I, b=I^{-3 / 2}, c=I / \sqrt{2 \pi \ln I}$ and $m_{k}^{(0)}=I^{k(k-1) / 2}$.

When $I=1$ (or $\alpha=\infty$ ) the system is monodisperse $\left(h_{0}(\sigma)=\delta(\sigma-1)\right.$ ) and its phase diagram consists of the usual vdW-binodal ending in the vdW critical point $\left(\eta_{c}=1 / 3, t_{c}=\right.$ $32 / 27$ ). For a modest polydispersity, say $I=1.02$ or $\alpha=50$, the phase diagram is similar to that of Fig. 4 of ref.8. For each parent-phase density $\eta_{0}$, there now is a different binodal. Each binodal is truncated upwards at a maximum temperature, $t_{m}$, with the corresponding densities, $\eta_{1}\left(t_{m}\right)$ and $\eta_{2}\left(t_{m}\right)$, lying respectively on the cloud-point and shadow curves. For a critical value of $\eta_{0}, \eta_{0}=\eta_{c}$, the corresponding binodal passes through the intersection of the latter two curves. This occurs for $t=t_{c}$ and, since, $\eta_{1}\left(t_{c}\right)=\eta_{2}\left(t_{c}\right)$, the point $\left(\eta_{c}, t_{c}\right)$ is a critical point where the two coexisting phases (1 and 2) become identical. However, in contradistinction with the monodisperse case $(I=1)$ the critical temperature is not the borderline between the one-phase and two-phase regions. Indeed, in the polydisperse case, the two-phase region extends to $t_{m}^{\prime}>t_{c}$, where $t_{m}^{\prime}$ corresponds to the maximum of the cloudpoint and shadow curves. At the same time the critical point loses one of its attributes, i.e. for $I \neq 1$, there also appears a second high-density critical point. The latter is polydispersityinduced 8 but, as will be shown below, not necessarily thermodynamically stable. When the 
polydispersity index $I$ is increased still further the ordinary low-density (vdW) critical point moves to a higher density and a lower temperature, while the polydispersity-induced highdensity critical point moves to a lower density and higher temperature, until for a limiting value of $I$, say $I=I^{*}$, the two critical points merge as illustrated in Fig.1. For $I>I^{*}$ there are no critical points and the phase behavior loses all contact with its monodisperse $(I=1)$ counterpart.

\section{TRUNCATED PARENT-PHASE SIZE-DISTRIBUTION}

For a polydisperse system the input data for the study of its phase behavior involve, besides the temperature $(t)$ and the average density $\left(\eta_{0}\right)$, the parent-phase size-distribution $h_{0}(\sigma)$ (as in ref.8 the subscript zero refers to the parent-phase). In theoretical work the values of $\sigma$ are usually distributed over the full interval of all possible sizes, $0 \leq \sigma \leq \infty$, whereas in the samples used in the laboratory these sizes usually span a continuous but finite interval, $\sigma^{\star} \leq \sigma \leq \sigma^{\star \star}$, with $0<\sigma^{\star}<1$ and $1<\sigma^{\star \star}<\infty$ if $\sigma=1$ represents the reference size (usually taken to be the average size). As stated already in ref.8, the small-size $\left(0<\sigma<\sigma^{\star}\right)$ and the large size $\left(\sigma^{\star \star}<\sigma<\infty\right)$ tails of $h_{0}(\sigma)$ will be harmless for strongly peaked distributions, i.e. for small polydispersities, but, as stated in the Introduction, they could lead to artifacts for large polydispersities characterized by broad size-distributions. Before studying the phase behavior of our system in the region of the (large) limiting polydispersity, $I \approx I^{*}$, where any contact with the monodisperse system is lost, we will first assess the influence of these tails on the results of ref.8. To this end we again consider the log-normal distribution of section $\mathbb{1}$ but normalize it now over the finite interval $\left(\sigma^{\star}, \sigma^{\star \star}\right)$ and rescale $\sigma$ in such a manner that the average value of $\sigma$ computed over this finite interval be again equal to one, i.e. the reference particle remains unchanged. In Fig.2 we show the effect of this truncation of the $\sigma$-domain on the form of $h_{0}(\sigma)$ for some representative values of $\left\{\sigma^{\star}, \sigma^{\star \star}\right\}$. In Table 1 we show its influence on the thermodynamic data. The overall effect of this truncation is hence to render the system less polydisperse. It also shifts the coexistence densities to lower values. Since some of our coexisting phases have densities for which the fluid phases could become metastable with respect to solid phases, a truncation of $h_{0}(\sigma)$ could render these fluid phases their thermodynamic stability. In any case, a quantitative comparison with experimental results will require phase diagrams calculated for truncated size-distributions and hence require information about $\sigma^{\star}$ and $\sigma^{\star \star}$. Since, however, no qualitative differences (or artifacts) are found, we will continue henceforth with the "untruncated" $h_{0}(\sigma)$ log-normal distribution, as defined at the end of section 【1 .

\section{THREE-PHASE EQUILIBRIA: LOCAL STABILITY}

As seen in section [I], for a sufficiently large polydispersity index $\left(I>I^{\star}\right)$ the phase behavior of our system loses any contact with its monodisperse counterpart. This is hence a good region to look for novel features such as three-phase equilibria. In the present section we will investigate how an initial (untruncated) log-normal parent-phase size-distribution,

$h_{0}(\sigma)$, fractionates into (two or three) daughter phases when its polydispersity index $I$ lies 
on either side of the limiting value $I^{*}\left(I^{*}=1.072\right.$ or $\left.\alpha^{*}=13.72\right)$ where the critical points disappear. We will focus on two cases: $\alpha=15$ or $I=1.066$ and $\alpha=13.5$ or $I=1.074$.

\section{A. Two-phase equilibria}

As discussed in detail in ref.8, these can be obtained by solving the two-phase coexistence conditions:

$$
p\left(T,\left[\rho_{1}\right]\right)=p\left(T,\left[\rho_{2}\right]\right) ; \mu\left(\sigma, T,\left[\rho_{1}\right]\right)=\mu\left(\sigma, T,\left[\rho_{2}\right]\right)
$$

where $\left\{\rho_{1}(\sigma)=\rho_{1} h_{1}(\sigma), \rho_{2}(\sigma)=\rho_{2} h_{2}(\sigma)\right\}$ are the density distributions of the two daughterphases resulting from the fractionation of the parent-phase, $\rho_{0}(\sigma)=\rho_{0} h_{0}(\sigma)$. The integral equation resulting from (6) can be solved as explained in ref.8. For the large $I$-values considered here we find two sets of binodals, each one being linked to a different critical point (see section [II). Since at these critical points the system is only marginally stable, i.e. we have $\delta^{2} f(T,[\rho])=0$, we first inquire for the local stability $\left(\delta^{2} f>0\right)$ of these solutions with respect to an infinitesimal change $\delta \rho(\sigma)$ of $\rho(\sigma)=\rho_{n}(\sigma)(n=1,2)$ :

$$
\delta^{2} f=\int d \sigma_{1} \int d \sigma_{2} \frac{\delta^{2} f(T,[\rho])}{\delta \rho\left(\sigma_{1}\right) \delta \rho\left(\sigma_{2}\right)} \delta \rho\left(\sigma_{1}\right) \delta \rho\left(\sigma_{2}\right)>0
$$

(see ref.8 for the explicit form of $\delta^{2} f$ ). It turns out that, for the $\eta_{0}$-values investigated, one of the two sets is locally unstable, i.e. it does not satisfy (7) (see Fig.3).

\section{B. Three-phase equilibria}

Similarly, the phase equilibria between three fluid-phases are governed by the solutions of :

$$
\begin{array}{r}
p\left(T,\left[\rho_{1}\right]\right)=p\left(T,\left[\rho_{2}\right]\right)=p\left(T,\left[\rho_{3}\right]\right) \\
\mu\left(\sigma, T,\left[\rho_{1}\right]\right)=\mu\left(\sigma, T,\left[\rho_{2}\right]\right)=\mu\left(\sigma, T,\left[\rho_{3}\right]\right)
\end{array}
$$

where $\left\{\rho_{1}(\sigma)=\rho_{1} h_{1}(\sigma), \rho_{2}(\sigma)=\rho_{2} h_{2}(\sigma), \rho_{3}(\sigma)=\rho_{3} h_{3}(\sigma)\right\}$ are the density distributions of the three daughter-phases. To solve (\$) we can generalize the method used in ref.8. The $h_{n}(\sigma)(n=1,2,3)$ are constrained by the particle number conservation of species $\sigma, x_{1} h_{1}(\sigma)+x_{2} h_{2}(\sigma)+x_{3} h_{3}(\sigma)=h_{0}(\sigma)$, whereas the total particle number conservation implies $x_{1}+x_{2}+x_{3}=1, x_{n}$ being the fraction of the parent-phase particles which went into phase $n$ (see ref.8 for details). The $\rho_{n}$ are constrained by the conservation of the total volume, $x_{1} v_{1}+x_{2} v_{2}+x_{3} v_{3}=v_{0}$, with $v_{n}=1 / \rho_{n}(n=0,1,2,3)$. This leaves us with two independent distributions, say $h_{1}(\sigma)$ and $h_{2}(\sigma)$, two independent number fractions, say $x_{1}$ and $x_{2}$, and two independent number densities, say $\rho_{1}$ and $\rho_{2}$. Given $\left(x_{1}, x_{2}\right)$ and $\left(\rho_{1}, \rho_{2}\right)$, the two distributions, $h_{1}(\sigma)$ and $h_{2}(\sigma)$, can be found by solving the system of two integral equations resulting from $\mu\left(\sigma, T,\left[\rho_{1}\right]\right)=\mu\left(\sigma, T,\left[\rho_{2}\right]\right)$ and $\mu\left(\sigma, T,\left[\rho_{2}\right]\right)=\mu\left(\sigma, T,\left[\rho_{3}\right]\right)$. As in ref.8, these two equations can be rewritten as: $h_{1}(\sigma)=h_{0}(\sigma) H_{1}(\sigma)$ and $h_{2}(\sigma)=$ $h_{0}(\sigma) H_{2}(\sigma)$, so that the normalization of the $h_{n}(\sigma)$ can be expressed as, $1=\int d \sigma h_{0}(\sigma) H_{1}(\sigma)$ 
and $1=\int d \sigma h_{0}(\sigma) H_{2}(\sigma)$, whereas solving the latter two equations together with, $p\left(T,\left[\rho_{1}\right]\right)=$ $p\left(T,\left[\rho_{2}\right]\right)$ and $p\left(T,\left[\rho_{2}\right]\right)=p\left(T,\left[\rho_{3}\right]\right)$ will determine $\left(x_{1}, x_{2}\right)$ and $\left(\rho_{1}, \rho_{2}\right)$. The full solution of a three-phase equilibrium problem for a polydisperse fluid is thus a rather complex task (hereby justifying some of the simplifying assumptions introduced above). In the case where the excess free-energy depends only on a finite number of moments of the distribution $h(\sigma)$, as is the case for the vdW free-energy, the above procedure can be simplified as the integral equations can then be transformed into a finite set of non-linear relations between these moments, as explained in ref.8. We found that the amount of labor involved is similar to that of the projection method of ref. 6 but contrary to the latter, the present method involves no approximation to the basic equations (6) or (8). In order to speed up the convergence of the solution method one may use the Powell-algorithm12 instead of the more traditional Newton-Raphson method 13 used in ref.8. An example of a three-phase coexistence found in this way is shown in Fig.4. We have verified that the case shown is locally stable, i.e. the $\rho_{n}(\sigma)(n=1,2,3)$ satisfy (7).

\section{RELATIVE STABILITY OF THE TWO-PHASE AND THREE-PHASE EQUILIBRIA}

As seen in the previous section, in strongly polydisperse systems it is possible to obtain, for the same input data, locally stable two-phase as well as three-phase solutions. This then raises the question of how to separate the stable from the metastable transitions, i.e. of the global stability of these solutions. All the globally stable states belong to the convex envelope of the free-energy surface. This envelope is such that the tangent plane through any of its points never cuts the free energy surface. Of course, in the polydisperse case these "surfaces" are defined in the infinite dimensional functional space supporting the density distributions, $\rho(\sigma)$. As a consequence, if $\rho_{e}(\sigma)$ belongs to the convex envelope of $f(T,[\rho])$ it must satisfy:

$$
f(T,[\rho]) \geq f\left(T,\left[\rho_{e}\right]\right)+\int d \sigma \frac{\delta f\left(T,\left[\rho_{e}\right]\right)}{\delta \rho_{e}(\sigma)}\left(\rho(\sigma)-\rho_{e}(\sigma)\right)
$$

for any $\rho(\sigma)$. It is of course not possible to verify this infinite number of conditions but, in practice, it will suffice to verify (9) for those states $\rho_{e}(\sigma)$ which are candidates for a stable equilibrium, i.e. for the solutions of eqs. (6) or (8), and for those states $\rho(\sigma)$ for which the distance between the free-energy surface and the tangent plane through $\rho_{e}(\sigma)$ is extremal, i.e. for the $\rho(\sigma)$ solution of:

$$
\frac{\delta f(T,[\rho])}{\delta \rho(\sigma)}=\frac{\delta f\left(T,\left[\rho_{e}\right]\right)}{\delta \rho_{e}(\sigma)}
$$

which usually amount to a finite number 6 for each $\rho_{e}(\sigma)$. In doing so, it is of course still possible that there exist (in the same parameter region) higher-order equilibria involving four, five, etc. phases which could still invalidate (9), but in view of the tremendous complexity of the global stability problem, we will limit ourselves here to the relative stability of the two-phase and three-phase equilibria found thus far. As a result of this search we find that the locally stable two-phase equilibrium solution of (6) is globally stable between an upper 
$\left(t_{+}\right)$and a lower $\left(t_{-}\right)$temperature. Above the upper temperature $\left(t>t_{+}\right)$the parent-phase is stable, while for $t<t_{+}$the parent -phase first fractionates into two phases while for $t<t_{-}$ the system further fractionates into three phases. Two examples of such phase diagrams are shown in Fig.5 and Fig.6.

\section{CONCLUSIONS}

The investigation of the phase behavior of the polydisperse generalization of the $\mathrm{vdW}$ free-energy which was started in ref.8 for relatively modest polydispersities $(I \approx 1.02)$ has been extended here to larger polydispersities $(I \approx 1.07)$. It is seen that when the polydispersity is increased the system's phase behavior gradually loses contact with its monodisperse counterpart. Indeed, for small polydispersities, the system investigated has two critical points, one of which is the polydisperse generalization of the $\mathrm{vdW}$ critical point, the other being polydispersity-induced. When the polydispersity is increased these two critical points merge for a limiting polydispersity $\left(I^{*} \approx 1.072\right)$. Above this threshold value $\left(I^{*}\right)$ there are no critical points. Similarly, when the polydispersity is increased, the two-phase region splits into two two-phase regions for the higher temperatures whereas for the lower temperatures a polydispersity-induced three-phase region appears. In order to determine the boundaries between these different regions of the phase diagram it is essential to investigate both the local and global stability of a large number of possible phase transitions. While some of these transitions are not even locally stable others are locally and globally stable. The situation quickly becomes very complex both physically and mathematically.

The phase behavior investigated here should be of relevance to the study of colloidal dispersions of spherical particles for which the size-distribution is monomodal. A detailed comparison will of course require a further extension of the present investigation towards

the solid phases, in particular their thermodynamic stability ${ }^{14}$ for the large polydispersities considered here.

\section{Acknowledgements}

M.B. acknowledges financial support from the F.N.R.S. 


\section{REFERENCES}

${ }^{1}$ R. Aris and G.R. Gavalas, Philos. Trans. R. Soc. London Ser.A 260, 351(1966); J.A. Gualtieri, J.M. Kincaid and G. Morrison, J. Chem. Phys. 77, 521(1982); J.J. Salacuse and G. Stell. J. Chem. Phys. 77, 3714(1982); J.B. Briano and E.D. Glandt, J. Chem. Phys. 80, 3336(1984).

${ }^{2}$ R.J. Hunter, Foundations of colloid science, (Clarendon Press, Oxford, 1993).

${ }^{3}$ W.B. Russel, D.A. Saville and W.R. Schowalter, Colloidal Dispersions, (Cambridge University Press, Cambridge, 1998); S. Chandrasekhar, Liquids Crystals, (Cambridge University Press, Cambridge, 1992); H.G. Elias, An introduction to Polymer Science, ( VCH Publishers, Inc., Weinhein, 1997).

${ }^{4}$ S. Leroch, G. Kahl and F. Lado, Phys. Rev. E 59, 6937(1999); S.E. Phan, W.B. Russel, J. Zhu and P.M. Chaikin, J. Chem. Phys., 108, 9789(1998); P. Bartlett, J. Chem. Phys. 107, 188(1997); R. McRae and A.D.J. Haymet, J.Chem.Phys. 88, 1114(1988); J.L. Barrat and J.P. Hansen, J. Physique 47, 1547(1986); and references therein.

${ }^{5}$ P. Bartlett and P.B. Warren, Phys. Rev. Lett. 82,1979(1999); P.B. Warren, Europhys. Lett. 46, 295(1999); J.A. Cuesta, Europhys. Lett. 46, 197(1999); R.M.L. Evans, Phys. Rev. E 59 , 3192(1999); R.M.L. Evans, D.J. Fairhurst and W.C.K. Poon, Phys. Rev. Lett. 81,1326(1998); P.B. Warren, Phys. Rev. Lett. 80, 1369(1998); R.P. Sear, Europhys. Lett. 44, 531(1998); P. Sollich and M.E. Cates, Phys. Rev. Lett. 80, 1365(1998); and references therein.

${ }^{6}$ P. Sollich, P.B. Warren and M. E. Cates, Adv. Chem. Phys. 116, 265 (2001).

${ }^{7}$ H. Xu and M. Baus, Phys. Rev. E 61, 3249(2000).

${ }^{8}$ L. Bellier-Castella, H. Xu and M. Baus J.Chem.Phys. 113, 8337(2000).

${ }^{9}$ M.R. Stapleton, D.J. Tildesley and N. Quirke, J. Chem. Phys. 92, 4456(1990); D.A. Kofke and E.D. Glandt, J. Chem. Phys. 90, 4456(1988).

${ }^{10}$ J.A. Gualtieri, J.M. Kincaid and G. Morrison, J. Chem. Phys. 77, 521(1989).

${ }^{11}$ A. Daanoun, C.F. Tejero and M. Baus, Phys. Rev. E 50, 2913(1994); T. Coussaert and M. Baus, Phys. Rev. E 52, 862(1995); A. Oukouiss and M. Baus, Phys. Rev. E 55, 7242(1997); R. Achrayah and M. Baus, Phys. Rev. E 57, 4361(1998); R. Lovett and M. Baus, J. Chem. Phys. 111, 5544(1999).

${ }^{12}$ M.J.D. Powell, in Numerical Methods for Nonlinear Algebraic Equations, edited by P. Rabinowitz (Gordon and Breach, New York, 1970).

${ }^{13}$ Numerical Recipes, ed. by W.H. Press, B.P. Flannery, S.A. Teukolsky, W.T. Vetterling (Cambridge University Press, Cambridge, 1989).

${ }^{14}$ P.A. Monson and D.A. Kofke, Adv. Chem. Phys. 115, 113(2000) and references therein. 


\section{Figure Captions}

FIG. 1. The low-density (circles) and high density (squares) critical points versus the polydispersity $\left(I=1+\frac{1}{\alpha}\right)$. The limiting value of $\alpha$ below which the two critical points disappear is $\alpha^{\star}=13.72\left(I^{\star}=1.073\right)$. Fig. 1a (resp. Fig. 1b) displays $\eta_{c}\left(\right.$ resp. $\left.t_{c}\right)$ versus $\alpha$.

FIG. 2. The full log-normal size distribution (dashed-dotted line) compared to a log-normal distribution truncated for $\sigma<\sigma^{\star}$ and $\sigma^{\star \star}<\sigma$ (full line). Both distributions are normalized $\left(m_{0}=1=m_{0}^{t}\right)$ and have the same average $\sigma$-value $\left(m_{1}=1=m_{1}^{t}\right)$. The case shown here corresponds to $\alpha=15(I=1.067)$ and $\sigma^{\star}=0.72, \sigma^{\star \star}=1.33$.

FIG. 3. Two sets of binodals for $\alpha=15$ and $\eta_{0}=0.68$. The full-line binodals correspond to locally stable solutions of eq.(6) while the dotted-line binodals are locally unstable. Also represented (diamond) is the second (unstable) critical point.

FIG. 4a A three-phase equilibrium for $\alpha=15$ and $\eta_{0}=0.506$ corresponding to a locally stable solution of eq.(8). The coexisting densities at $t=1.07$ are $\eta_{1}=0.284, \eta_{2}=0.652$ and $\eta_{3}=0.930$.

FIG. 4b Coexisting distributions $h_{i}(\sigma)(i=1$ : full-line, $i=2$ : dotted-line and $i=3$ : dashed-dotted line) at $t=1.07$ and for the $\alpha$ and $\eta_{0}$ values of Fig. 4 a.

FIG. 5 A phase diagram for $\alpha=15$ and $\eta_{0}=0.506$. The one-phase region corresponds to $t>t_{+} \simeq 1.75$, the two-phase region to $t_{+}<t<t_{-} \simeq 1.24$, while the three-phase region corresponds to $t<t_{-}$.

FIG. 6 The same as Fig. 5 but for $\alpha=13.5$ and $\eta_{0}=0.506$. Here $t_{+} \simeq 1.75$ and

$t_{-} \simeq 1.26$. Note that while in Fig. 5 the new (third) phase is a low-density phase here it is an intermediate-density phase. 


\section{TABLES}

\begin{tabular}{|c|c|c|c|}
\hline$\sigma^{\star}$ & $\sigma^{\star \star}$ & $\eta_{1}$ & $\eta_{2}$ \\
\hline 0 & $\infty$ & 0.24 & 0.69 \\
\hline 0.30 & 2.75 & 0.24 & 0.69 \\
\hline 0.48 & 1.76 & 0.23 & 0.68 \\
\hline 0.56 & 1.54 & 0.20 & 0.66 \\
\hline 0.70 & 1.32 & 0.15 & 0.63 \\
\hline
\end{tabular}

TABLE I. Typical shifts in the coexisting densities of the low-density $\left(\eta_{1}\right)$ and high density $\left(\eta_{2}\right)$ phase for $t=1, \alpha=15$ after truncation of the log-normal parent-phase size-distribution for $\sigma<\sigma^{\star}$ and $\sigma^{\star \star}<\sigma$. The corresponding monodisperse results are $\eta_{1}=0.10, \eta_{2}=0.61$. 
Fig. 1a, Bellier-Castella et al., JCP

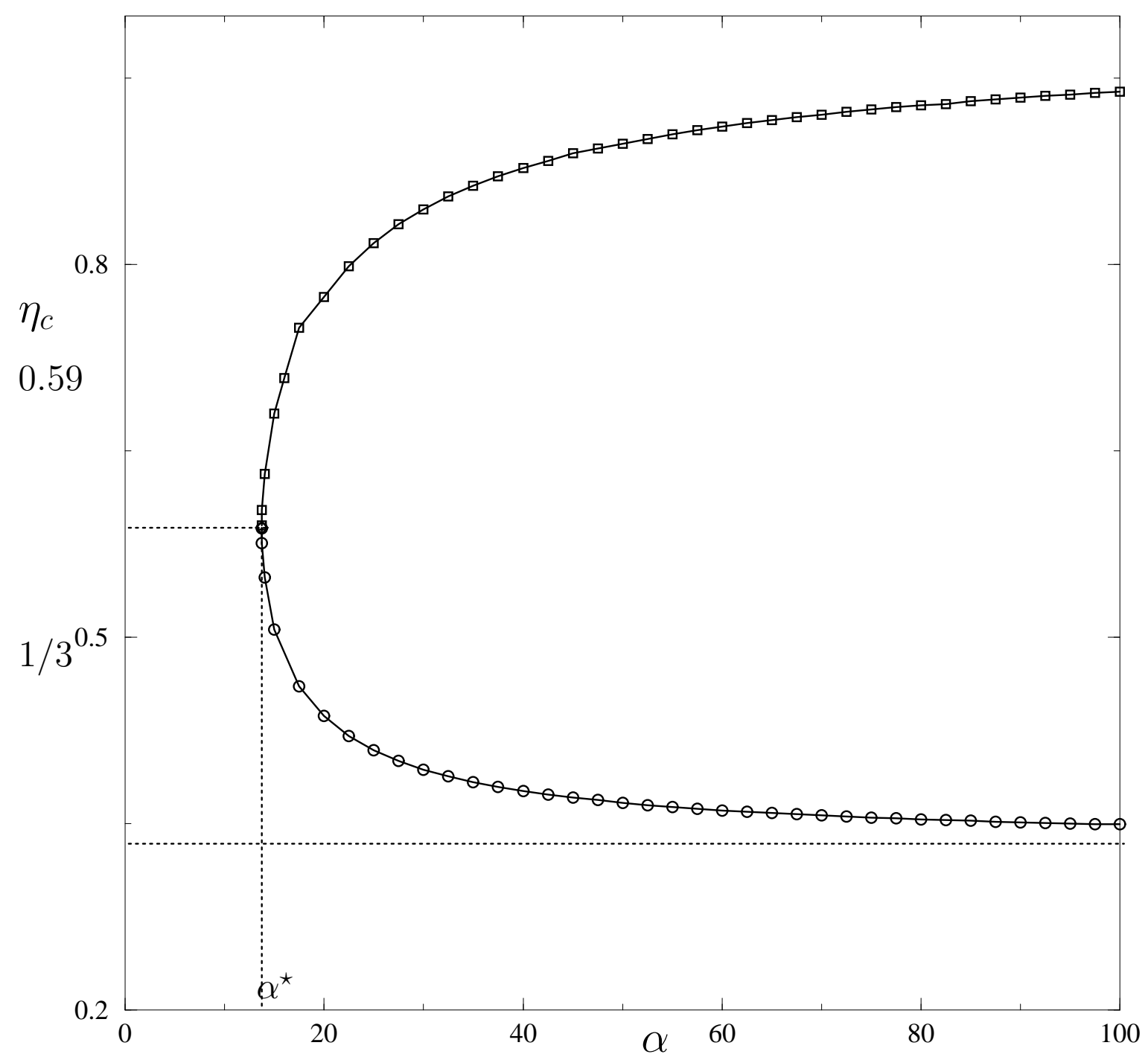


Fig. 1b, Bellier-Castella et al., JCP

1.18

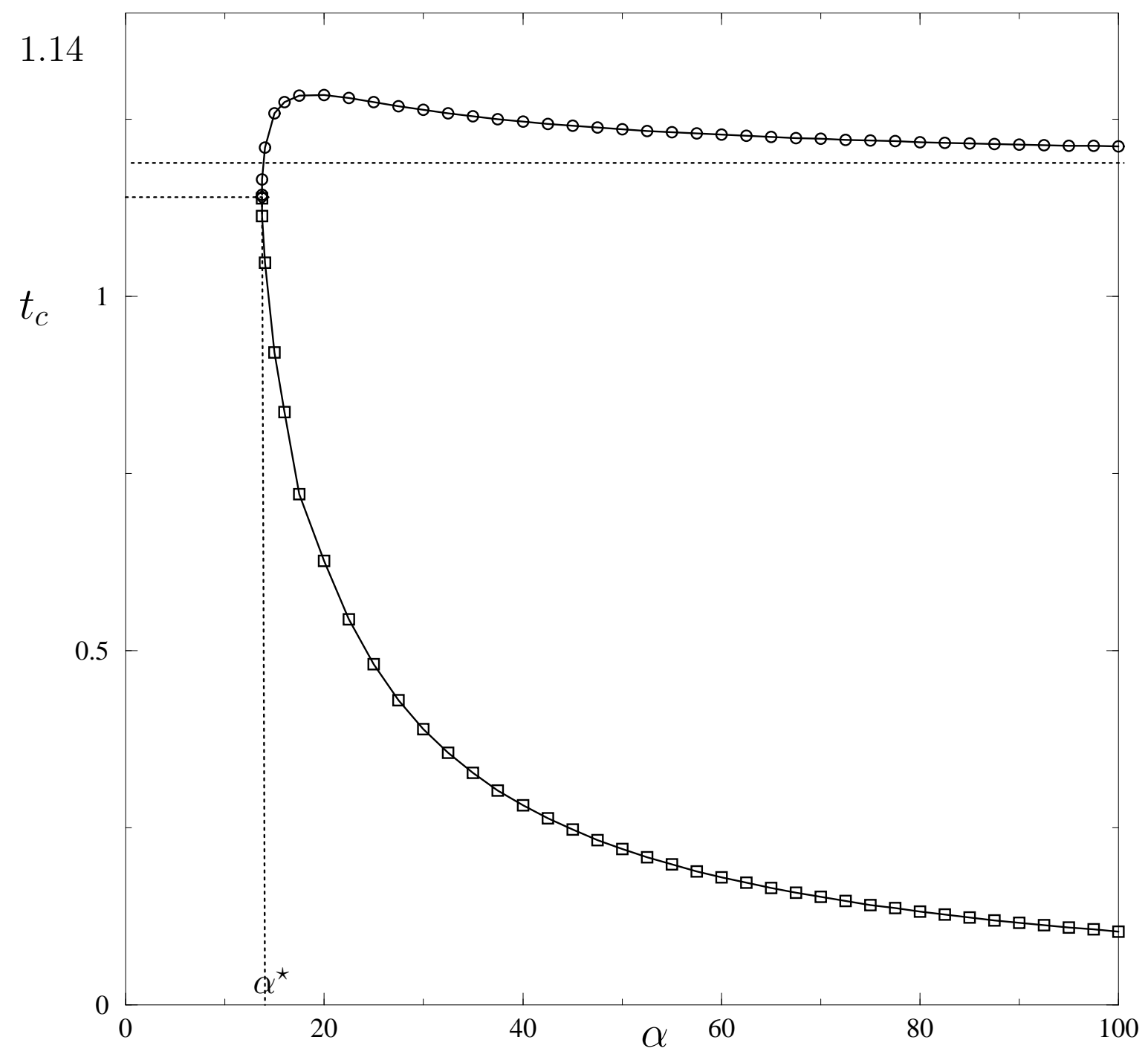


Fig. 2 , Bellier-Castella et al., JCP

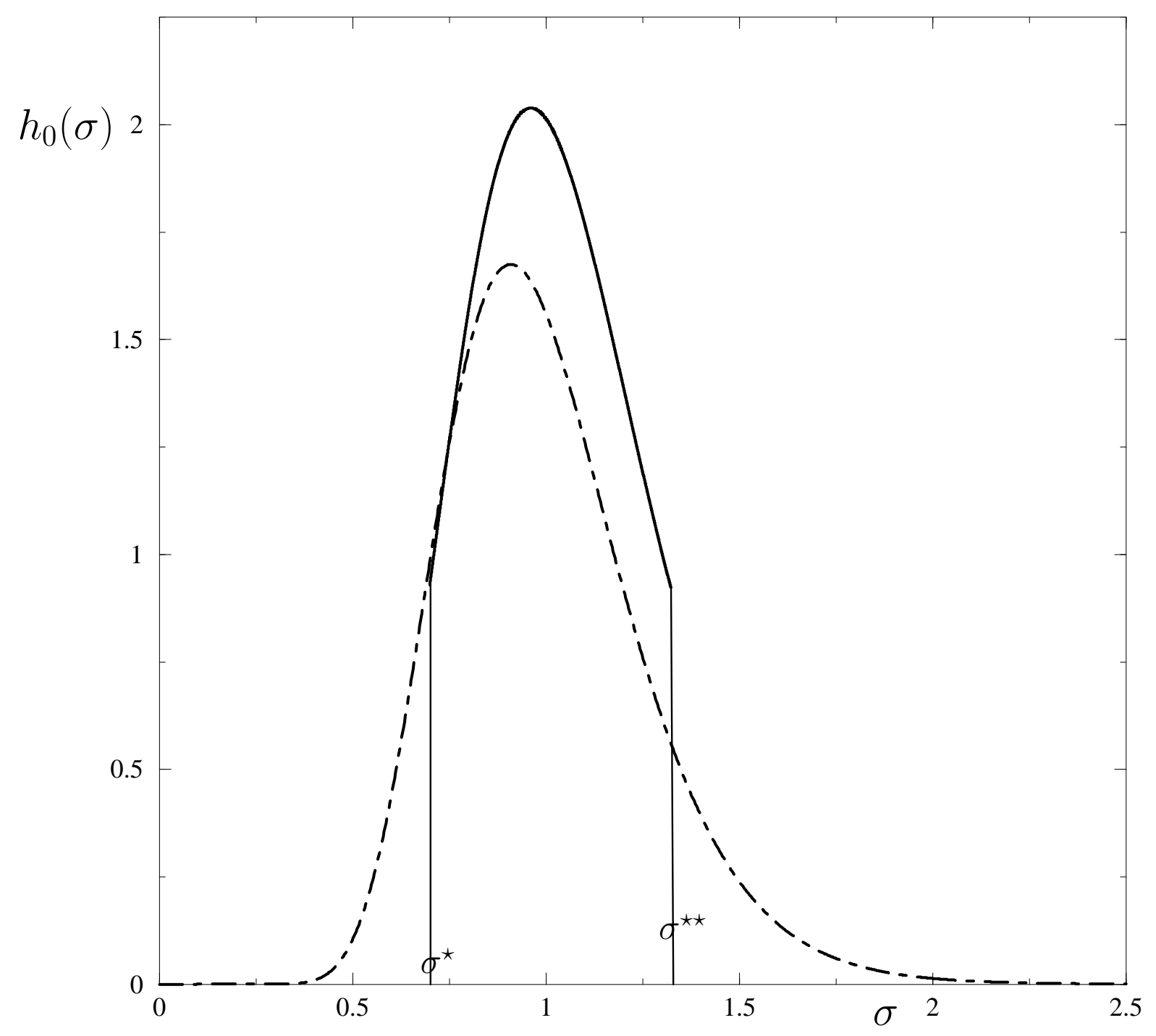


Fig. 3, Bellier-Castella et al., JCP

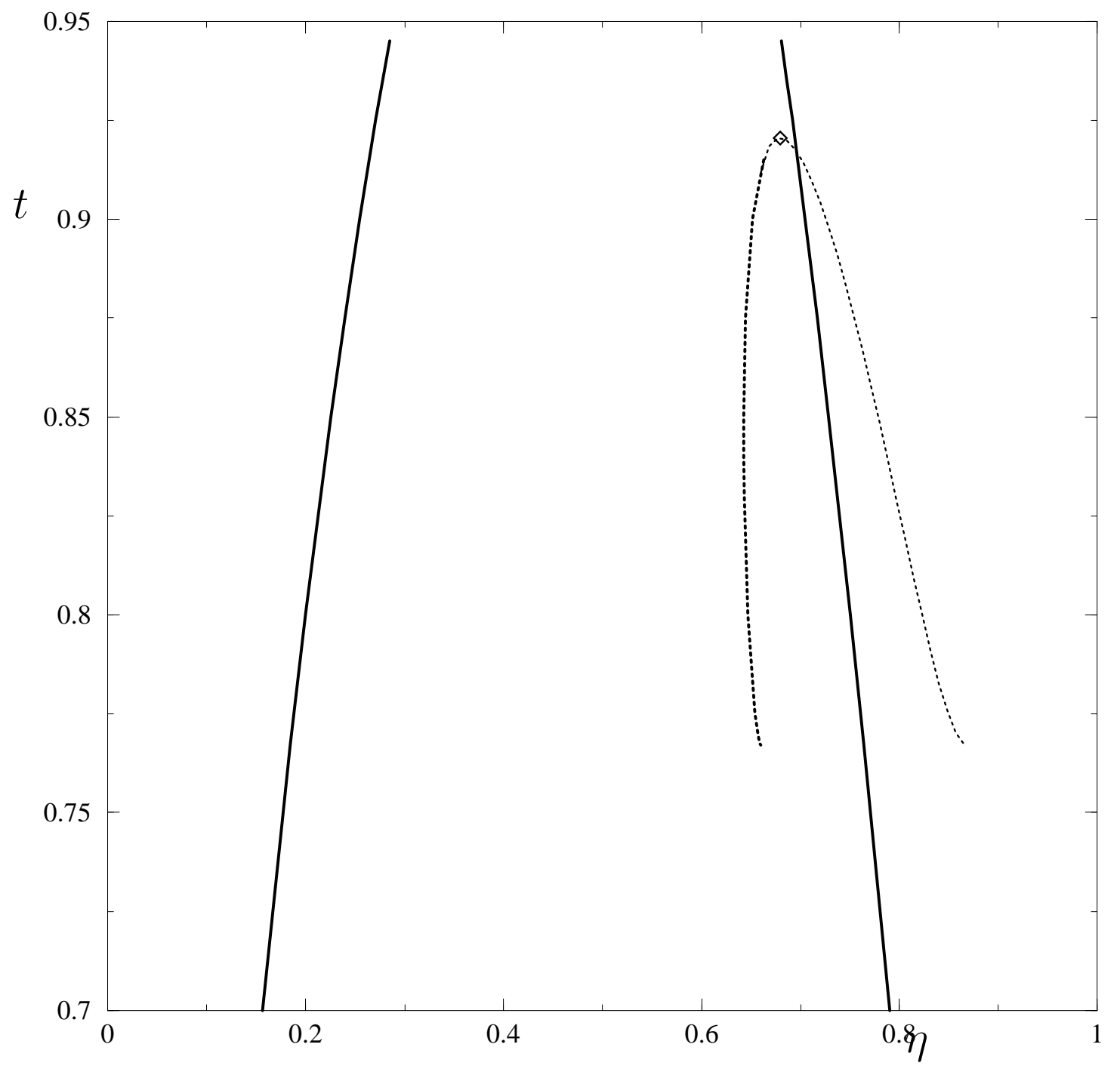


Fig. 4a , Bellier-Castella et al., JCP

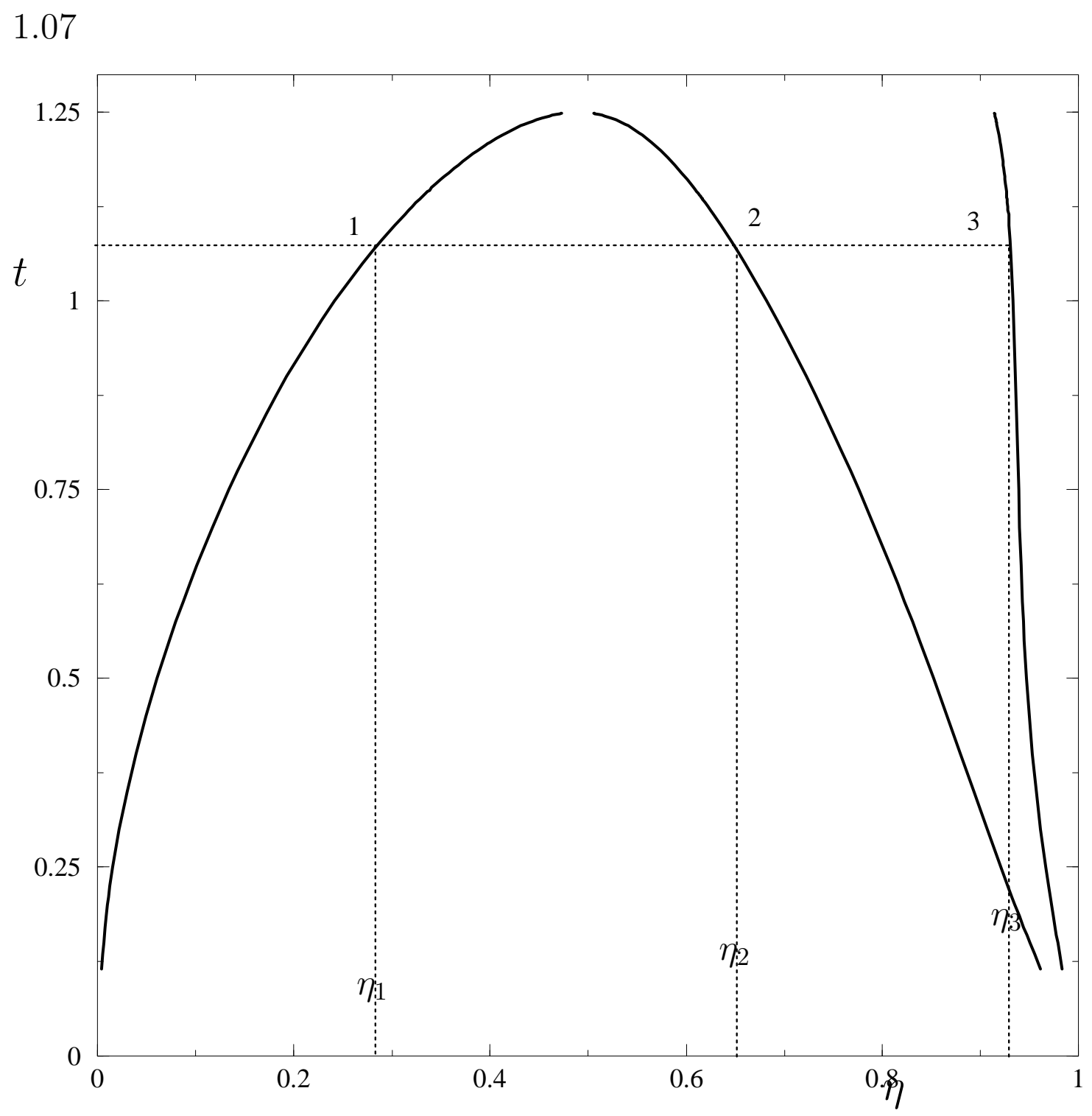


Fig. 4b, Bellier-Castella et al., JCP

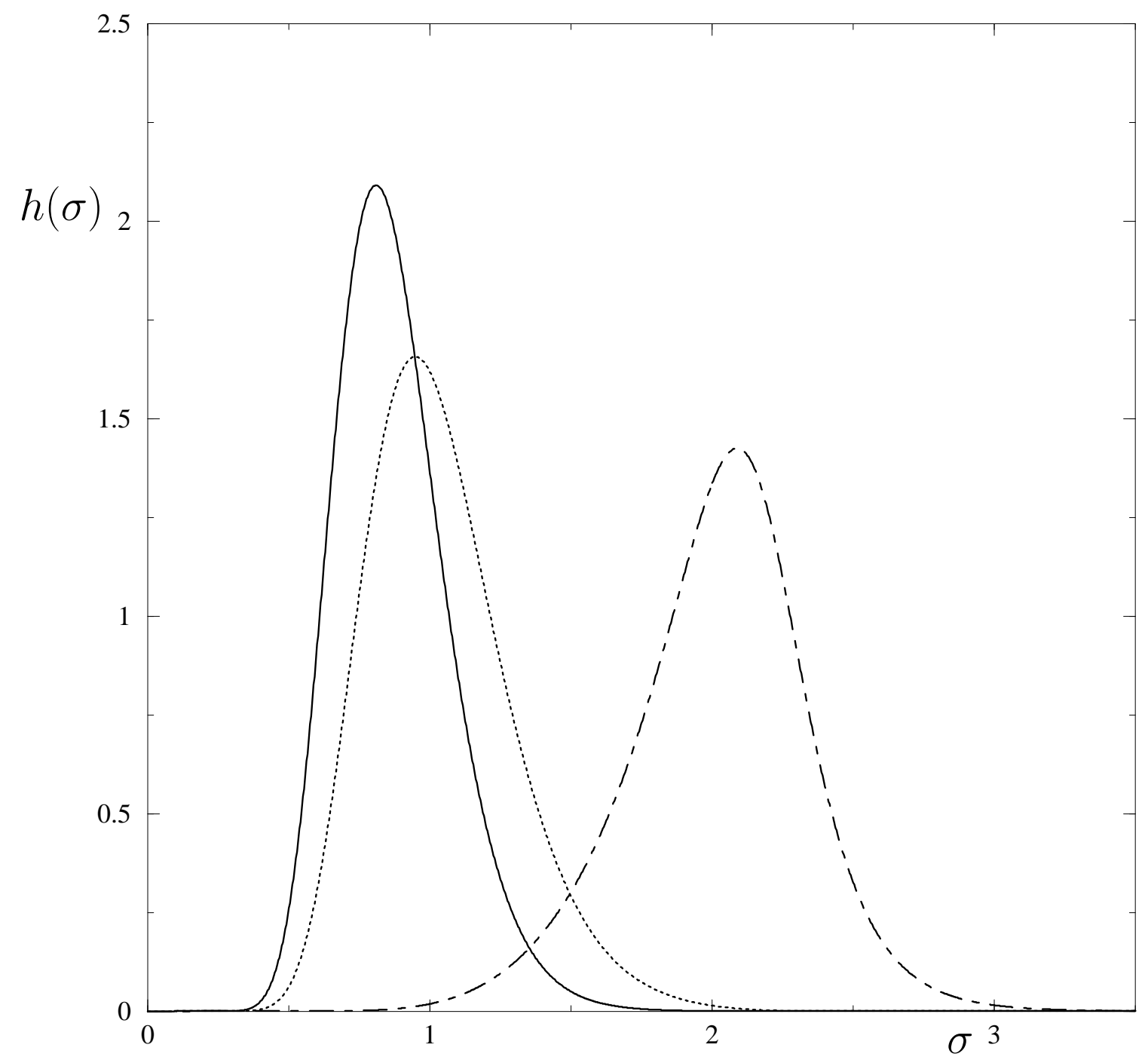


Fig. 5, Bellier-Castella et al., JCP

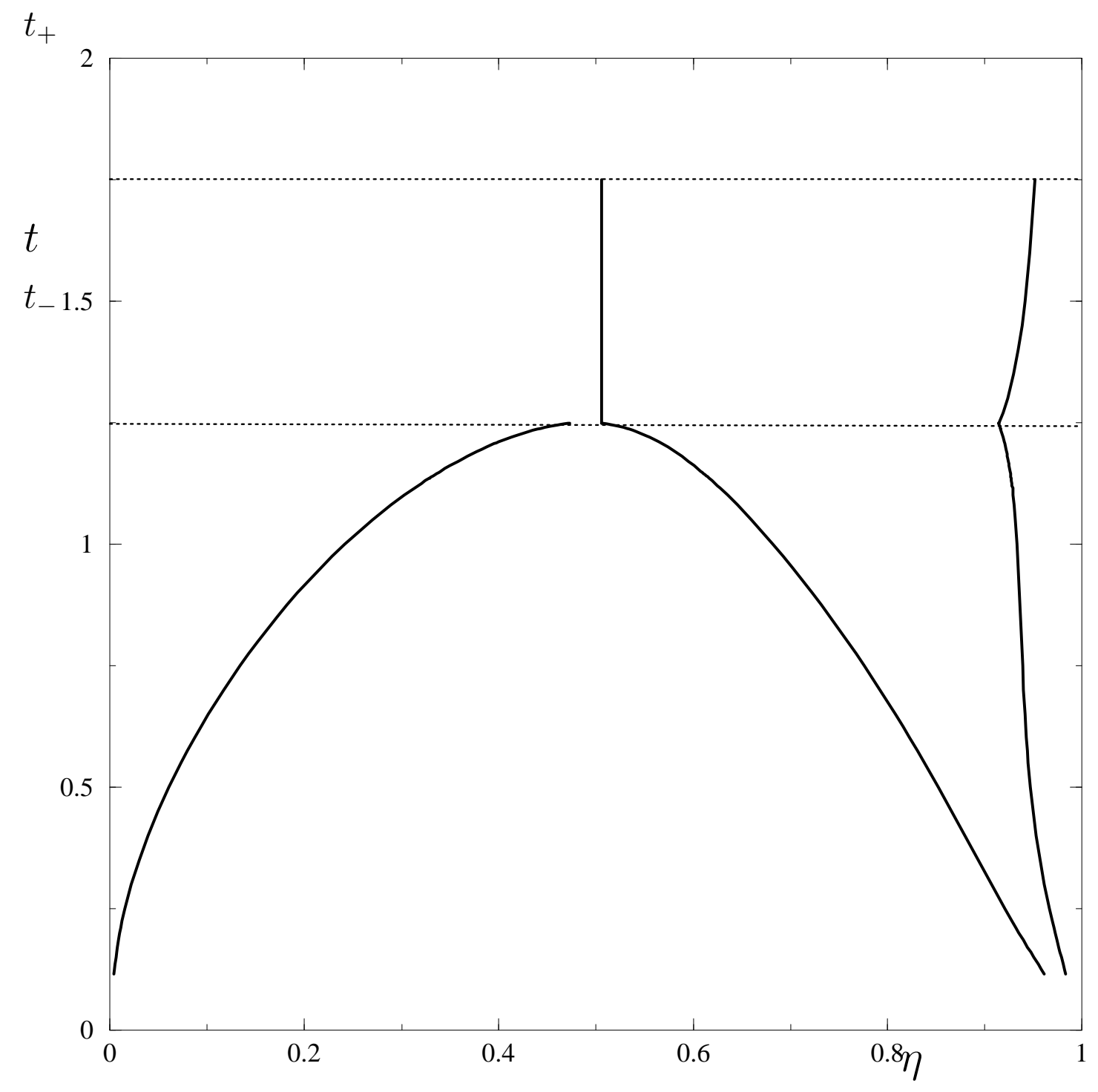


Fig. 6 , Bellier-Castella et al., JCP

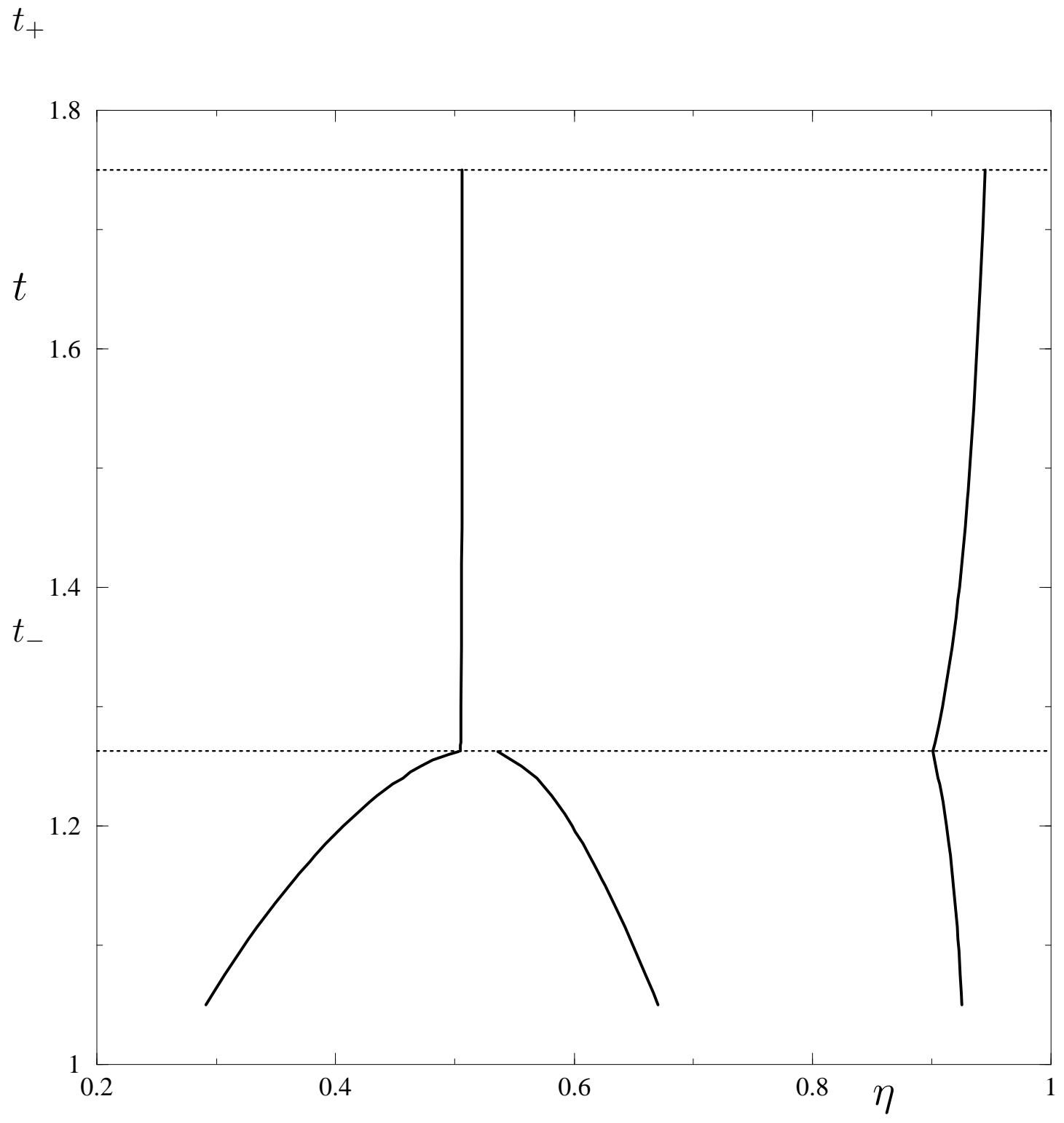

